Revista Española de

Cirugía Oral y

Maxilofacial

www.elsevier.es/recom

\title{
Original
}

\section{Distracción ósea: tratamiento de la apnea obstructiva en neonatos con micrognatia}

\author{
Adoración Martínez Plaza ${ }^{a, *}$, Idelfonso Martínez Lara ${ }^{a}$, Blas García Medina ${ }^{a}$, Ricardo \\ Fernández Valadés ${ }^{b}$, Ana Belén Marín Fernández ${ }^{a}$ y Carlos Bailón Berrio ${ }^{a}$
}

aServicio de Cirugía Oral y Maxilofacial, Hospital Universitario Virgen de las Nieves, Granada, España

bServicio de Cirugía Pediátrica, Hospital Universitario Virgen de las Nieves de Granada, España

\section{INFORMACIÓN DEL ARTÍCULO}

Historia del artículo:

Recibido el 15 de febrero de 2011

Aceptado el 3 de mayo de 2011

Palabras clave:

Distracción mandibular osteogénica;

Hipoplasia mandibular;

Apnea obstructiva

\section{R E S U M E N}

Las malformaciones craneofaciales (secuencia de Pierre Robin, síndrome de ThreacherCollins, síndrome de Nager, etc.) con frecuencia van asociadas a hipoplasia mandibular grave, que puede causar obstrucción de la vía aérea superior por retroposición de la base de la lengua dentro del espacio faríngeo posterior.

La mayoría de los pacientes responden al tratamiento postural, en decúbito prono, puede ser necesario controlar la saturación de oxígeno, insertar un tubo nasofaríngeo e incluso intratraqueal. En casos más graves con pausas prolongadas y frecuentes de apnea, la traqueostomía puede ser necesaria, pero se asocia a una alta morbilidad y, ocasionalmente, mortalidad.

En los últimos 2 años, en la Unidad Multidisciplinaria de Labio y Fisura Palatina del Hospital Virgen de las Nieves de Granada, se ha tratado a 4 niños con apnea obstructiva grave secundaria a hipoplasia mandibular grave mediante distracción mandibular osteogénica, y este procedimiento se ha mostrado eficaz en la resolución del problema. Ha evitado la traqueostomía y se ha elongado la mandíbula en el plazo de 3-4 semanas. En este tiempo han desaparecido los problemas respiratorios obstructivos, así como también de la deglución, y los resultados estéticos obtenidos han resultado excelentes y las complicaciones, por el momento, mínimas.

(C) 2011 SECOM. Publicado por Elsevier España, S.L. Todos los derechos reservados.

Mandibular distraction: treatment of obstructive apnea in neonates with micrognathia

A B S T R A C T

Craniofacial malformations (Pierre-Robin sequence, Treacher-Collins syndrome, Nager syndrome, etc.) are frequently accompanied by severe mandibular hypoplasia, which can cause upper airway obstruction due to retroposition of the base of the tongue in the posterior pharyngeal space.

\footnotetext{
${ }^{*}$ Autor para correspondencia.

Correo electrónico: adoracionmartinez@medicalpur.es (A. Martínez Plaza).
} 
The majority of patients respond to postural treatment in decubitus prono. It may be necessary to monitor oxygen saturation and insert a nasopharyngeal or even an endotracheal tube. Tracheostomy may be necessary in more serious cases with long and frequent apnea pauses, but it is associated with high morbidity and occasional mortality. In the last two years, four children with severe obstructive apnea secondary to mandibular hypoplasia were treated by means of "osteogenic mandibular distraction" in the multidisciplinary Cleft Palate Department of Virgin de las Nieves Hospital (Granada, Spain). This procedure effectively resolved the problem, making tracheostomy unnecessary and lengthening the jaw within 3-4 weeks; in this period, obstructive respiratory problems and swallowing difficulties disappeared. The aesthetic results were excellent and the complications so far have been minimal. Objective: To present the results of a patient series with several types of POP treated using the same approach and operation.

○ 2011 SECOM. Published by Elsevier España, S.L. All rights reserved.

\section{Introducción}

La secuencia de Pierre Robin ${ }^{1}$, cuya incidencia es aproximadamente de 1/8.500 nacidos vivos ${ }^{2}$, se acompaña de micrognatia, glosoptosis y obstrucción respiratoria alta, y con frecuencia se asocia a fisura palatina. Puede presentarse aislada o asociada a otros síndromes (Tracher-Collins, Nager, velocardiofacial, etc. $)^{3}$. Estos niños pueden mostrar importantes dificultades para respirar y deglutir, a causa de la obstrucción de la vía aérea superior, debido a la disminución del espacio faríngeo por la retropulsión de la lengua 4 . En el 25\% de los pacientes con secuencia de Pierre Robin pueden aparecer dificultades en la alimentación, aun en ausencia de alteraciones de la respiración ${ }^{5}$.

Desde hace tiempo se han estado utilizando diversos tratamientos para solventar este grave problema. Tratamientos posturales, intubación nasofaríngea, glosopexia, etc., con lo que se intenta evitar la realización de una traqueostomía6-9. Tener que mantener esta traqueostomía largo tiempo se asocia a una morbilidad importante, además de una alteración de la adquisición del lenguaje e integración social ${ }^{10}$.

Hace unos años se comenzó a utilizar la distracción mandibular osteogénica para elongar la mandíbula, avanzar la base de la lengua con el agrandamiento del espacio hipofaríngeo y mejorar la obstrucción de la vía aérea ${ }^{11}$. Así se evita tener que realizar traqueostomía y se mejora la alimentación oral de estos niños, lo que contribuye a un mejor desarrollo ponderal y evita daños neurológicos a veces irreversibles. Ello hace que se considere esta técnica como la de elección para tratar a pacientes con apnea obstructiva por hipoplasia mandibular grave.

Las indicaciones para el tratamiento se hacen a partir de diversos estudios, entre los cuales el más relevante es la polisomnografía, en la que se detectan el número total de episodios de apnea en 1 hora (índice de apnea), número total de episodios de hipoxia u obstrucción parcial en una hora (índice de hipoxia) y la suma de ambos (índice respiratorio) ${ }^{12}$. La realización de una nasofibroscopia con respiración espontánea diferencia la obstrucción debida a una deficiencia mandibular, de la debida a una traqueomalacia ${ }^{3} \mathrm{u}$ otras alteraciones congénitas de las vías aerodigestivas superiores. Es posible analizar la anatomía mandibular mediante tomografía computarizada (TC) con reconstrucción tridimensional, para planificar el lugar de la osteotomía ${ }^{13}$ y el vector de distracción osteogénica. La valoración debe hacerla un equipo multidisciplinario que defina el problema anatómico y funcional para evitar una traqueostomía y prevenir sus secuelas a largo plazo ${ }^{14}$.

\section{Material y métodos}

Nuestra experiencia con la distracción mandibular temprana en pacientes con apnea obstructiva secundaria a hipoplasia mandibular grave incluye a un total de 4 pacientes. Dos casos presentaban secuencia de Pierre Robin, un caso, síndrome de Cornelia de Lange, y dos casos, síndrome de TreacherCollins.

Mediante un examen clínico diagnosticamos la hipoplasia mandibular grave y la insuficiencia respiratoria se observó mediante seguimiento de la saturación de oxígeno. También se evaluó la articulación temporomandibular, muchos pacientes micrognáticos presentan limitaciones de grado variable de la apertura bucal, relacionada directamente con alteraciones morfológicas del cóndilo, factores mecánicos secundarios a la poca longitud del cuerpo mandibular, o bien a algún grado de anquilosis de tipo extraarticular. En todos los casos, encontramos una apertura oral limitada por la escasa longitud del cuerpo y rama mandibular. Entre los estudios preoperatorios sistemáticos se realizó una TC tridimensional (fig. 1). La fibroscopia diagnóstica localizó el sitio de la obstrucción, que en todos los casos fue a nivel del espacio faríngeo posterior, con lo que se observó la retroposición lingual. También se tomaron fotos para documentar por completo los casos clínicos.

La técnica quirúrgica realizada en todos ellos fue la distracción mandibular osteogénica bilateral mediante el distractor externo unidireccional de Molina (Molina Uni-diret. Distractor: KLS Martín Group), tal como han descrito Molina et $\mathrm{al}^{15}$.

Con anestesia general, se realiza una incisión en la mucosa de la línea oblicua del área retromolar hacia el vestíbulo de aproximadamente $1,5 \mathrm{~cm}$, a fin de exponer el ángulo mandi- 


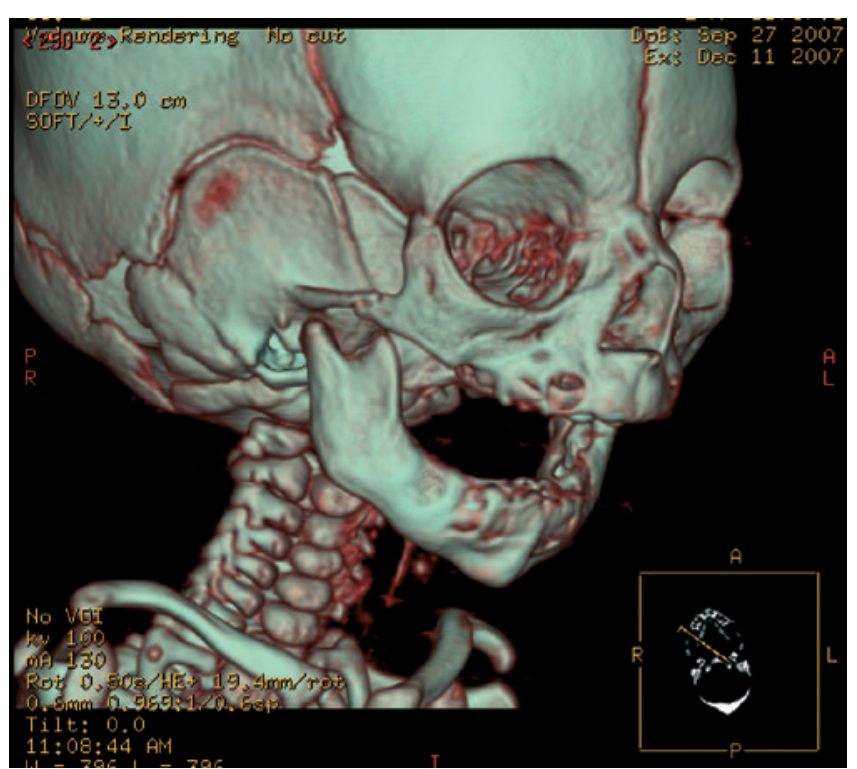

Figura 1 - Tomografía computarizada tridimensional. Hipoplasia mandibular grave.

bular y la porción inicial de la rama ascendente. La disección debe ser limitada para no lesionar la vascularización de la mandíbula ya hipoplásica. Con tinta se marca el sitio de la corticotomía, así como el lugar donde se van a insertar los clavos del distractor. Este momento es importante, ya que el lugar de inserción de los clavos va a determinar el vector de distracción, y su localización debe evitar dañar el nervio mandibular y los gérmenes dentarios.

La corticotomía debe ser una corticotomía "externa y extendida", que preserva la integridad del nervio, la vascularización intramedular y todos los gérmenes dentarios. Se realiza mediante una fresa de fisura y se inicia en el ángulo retromolar, primero seccionando los contrafuertes lateral y medial. Luego se extiende en toda la superficie lateral del ángulo mandibular en su porción más basal, donde el hueso es más duro y tiene más espesor. Cuando toda la corticotomía externa está hecha y se muestra el hueso esponjoso sangrante, el corte óseo se detiene. Se preserva unos milímetros de cortical interna, área que protege tanto al nervio como a la arteria mandibular. El vector de distracción perpendicular hace que la elongación sea mayor a nivel del ángulo y menor en el reborde alveolar, reproduciendo así los movimientos de rotación del crecimiento mandibular fisiológico. De este modo se aumenta la dimensión vertical de la rama, al mismo tiempo que se recrea el crecimiento mandibular en el sentido de las agujas del reloj. Respecto al vector de distracción deseado, el oblicuo fue el de elección, con lo que se produjo más neoformación ósea en el ángulo y menos en el alvéolo, lo cual elonga la rama ascendente y el cuerpo mandibular.

Todo ello se realiza de forma bilateral, y por último se insertan de forma percutánea 2 clavos a cada lado de corticotomía. Estos son siempre bicorticales, y mediante palpación digital debe controlarse la cara lingual de la mandíbula. Deben quedar muy estables para evitar una complicación relativamente frecuente, como es la pérdida de los clavos

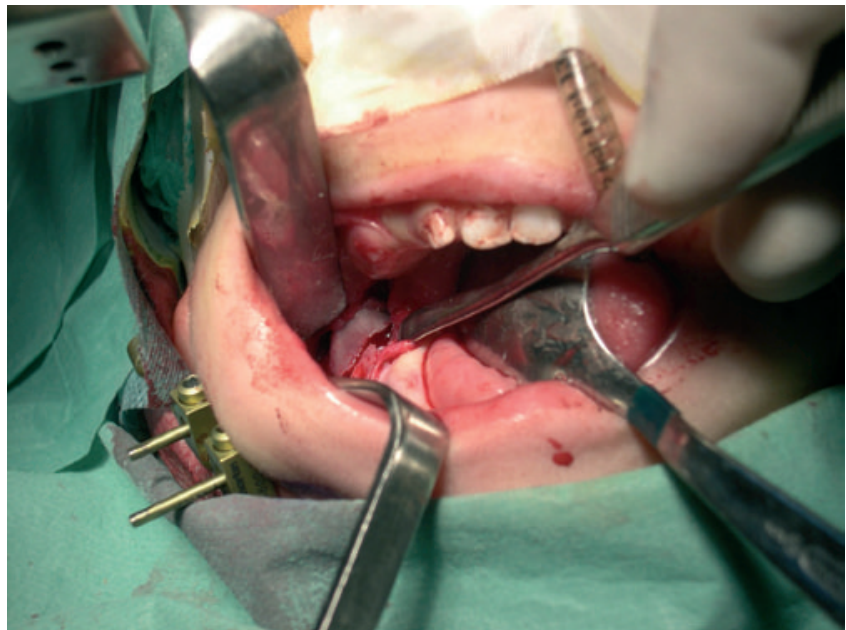

Figura 2 - Corticotomía en área retromalar. Inserción del distractor externo.

durante el proceso de distracción. La distancia de inserción de éstos a la corticotomía debe tener un mínimo de 3-5 mm. Para minimizar las cicatrices, debemos coger un pliegue de piel antes de introducir el segundo clavo para disminuir la presión cutánea (fig. 2).

Los clavos deben ser paralelos entre sí, para facilitar la introducción y la fijación del distractor. Se abren intraoperatoriamente de 3 a $5 \mathrm{~mm}$, y debe comprobarse que no hay ningún puente de cortical que impida la elongación mandibular. Después se desactivan hasta que comience el período activo de distracción. Se cierra la incisión con sutura reabsorbible.

El período de latencia es de 2-3 días y se inicia la distracción a ritmo de $1 \mathrm{~mm}$ al día, $0,5 \mathrm{~mm}$ cada $12 \mathrm{~h}$, con lo que inmediatamente se produce un avance sagital de la mandíbula y de la base de la lengua. El período de contención es de 6-8 semanas, una vez finalizada la distracción.

Los distractores se retiran fácilmente y para ello los pacientes no necesitan ingreso hospitalario.

\section{Casos clínicos}

Caso 1

Recién nacido con síndrome de Treacher-Collins, nacido a término con peso adecuado a la edad gestacional, cariotipo normal XY y fisura palatina, que ingresó en unidad neonatal 40 días por problemas respiratorios y de deglución. Fue dado de alta hospitalaria, y reingresó al mes por insuficiencia respiratoria. Durante el ingreso se realizó gastrostomía (imposibilidad de intubación por lo que se empleó mascarilla laríngea). Se dio alta al paciente y volvió a tener varios reingresos por problemas respiratorios que cada vez fueron más frecuentes. Con 16 meses se realizó distracción mandibular. Tras el período de distracción de 3 semanas $(17 \mathrm{~mm})$, desaparecieron los problemas respiratorios y de deglución. En un segundo tiempo se hizo el cierre de la fisura palatina mediante veloplastia intravelar sin incidencias. 


\section{Caso 2}

Recién nacida a término, con bajo peso (1.820 g), con síndrome de Cornelia de Lange y fisura palatina. Presentó episodios de insuficiencia respiratoria que se solucionaron inicialmente con medidas posturales, pero que condicionaron el ingreso en unidad de cuidados intensivos neonatales durante 3 meses. Con 3,5 meses se le realizó distracción mandibular y gastrostomía. Tras el período de distracción de 18 días (16 $\mathrm{mm})$, desaparecieron los problemas respiratorios y paulatinamente comenzó a deglutir, lo que permitió la retirada de la gastrostomía. Se reparó la fisura palatina con veloplastia intravelar a los 2 años sin complicaciones.

\section{Caso 3}

Recién nacido de 41 semanas de gestación y 3.230 g de peso, que precisó reanimación tras el parto. Presenta secuencia de Pierre Robin y síndrome malformativo, con asimetría torácica e implantación baja de pabellones auriculares (fig. 3). Antecedentes de hermano y tío paterno con secuencia de Pierre Robin. Cariotipo normal. Desde el nacimiento experimenta episodios de apnea obstructiva que se resolvieron con medidas posturales. Mejoría transitoria hasta que fue dado de alta, ya que los padres inicialmente no aceptaron tratamiento con distracción mandibular por tener experiencia con el hijo anterior que mejoró y no necesitó otro tratamiento que el postural. Tras el alta, el paciente presentó dos episodios de insuficiencia respiratoria grave y en uno de ellos precisó reanimación cardiopulmonar e intubación. Desde entonces presentó episodios frecuentes de apnea obstructiva alta que mejoraron con medidas posturales. Se realizó distracción mandibular a los 4 meses de edad. Tras la distracción con

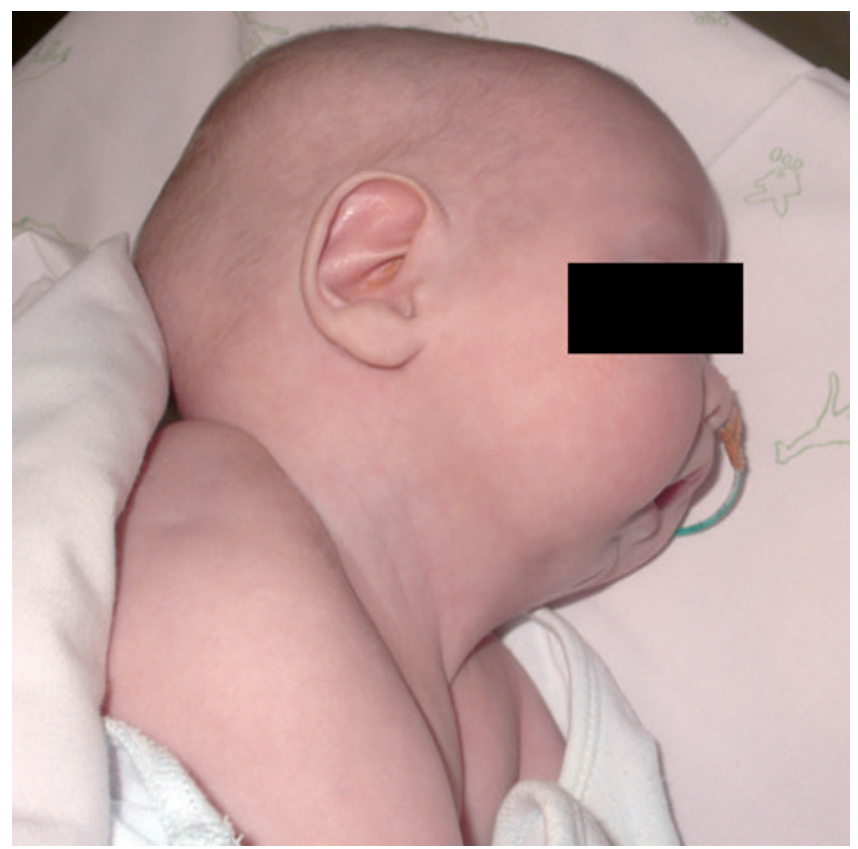

Figura 3 - Secuencia de Pierre Robin. Alimentación con sonda nasogástrica avance de $18 \mathrm{~mm}$, desapareció la sintomatología respiratoria y mejoró la deglución. Se ha cerrado la fisura con veloplastia intravelar sin complicaciones a los 20 meses de edad.

\section{Caso 4}

Recién nacido de 34 semanas de gestación y 1.960 g de peso, que presenta secuencia de Pierre Robin, también síndrome polimalformativo con escoliosis, artrogriposis, aneurisma septo interventricular, orejas de implantación baja, anomalías de posición e hipoplasia de mamilas. El cariotipo y el estudio genético molecular de síndrome de CATCH 22 son normales. No presentó ninguna lesión en la ecografía transfontanelar ni en la TC. Durante la observación presentó episodios aislados de desaturación que desaparecieron en decúbito lateral; sin embargo, cuando se intentó alimentación oral, se produjeron desaturaciones por debajo del $60 \%$, que se recuperaron tras dejar de tomar. Se instauró alimentación por sonda nasogástrica desde el primer momento. La nasofibroscopia sólo mostró la glosoptosis como posible causa de esta dificultad para la deglución. Tras no observar otra afección como causa de las alteraciones en la alimentación, se realizó distracción mandibular a los 4,5 meses de vida (fig. 4).

\section{Resultados}

Se trató a 4 pacientes entre los 3,5 y los 16 meses: dos con secuencia de Pierre Robin, uno con síndrome de ThreacherCollins y otro con síndrome de Cornelia de Lange. Se realizó distracción mandibular osteogénica unidireccional, con distractores externos de Molina. No hubo que someter a ninguno de los 4 pacientes a traqueostomía durante sus episodios de

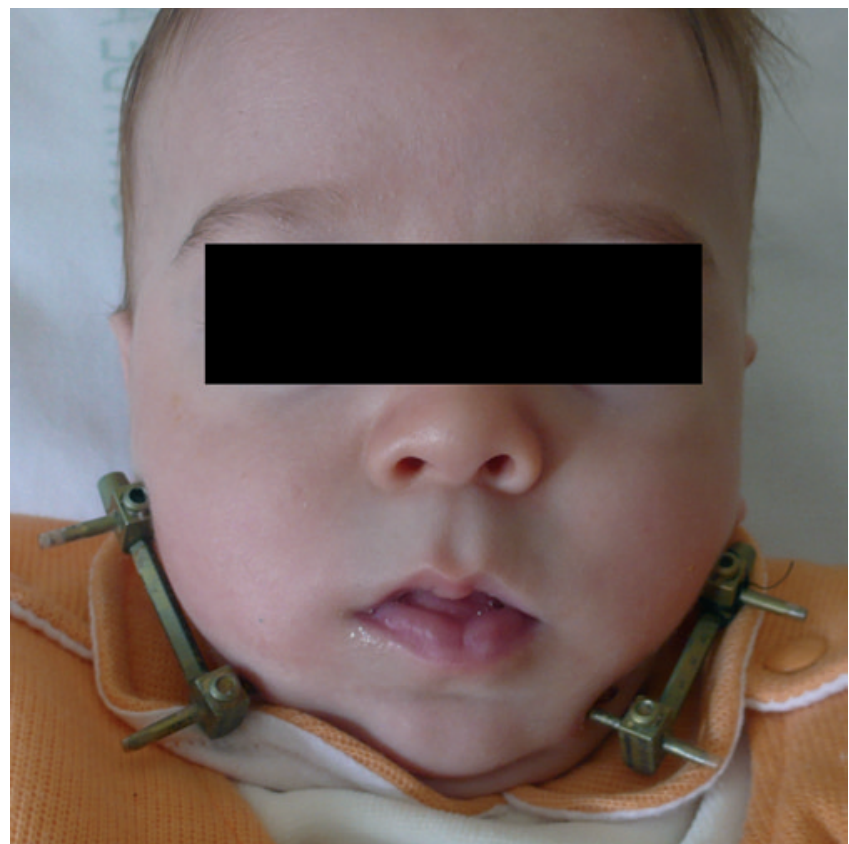

Figura 4 - Secuencia de Pierre Robin en período de distracción ósea. 
Tabla 1 - Resumen de los casos clínicos

\begin{tabular}{|c|c|c|c|c|}
\hline & $\begin{array}{l}\text { Síndrome de } \\
\text { Treacher-Collins }\end{array}$ & $\begin{array}{l}\text { Síndrome de } \\
\text { Cornelia de Lange }\end{array}$ & $\begin{array}{c}\text { Síndrome de } \\
\text { Pierre Robin }+ \\
\text { polimalformativo }\end{array}$ & $\begin{array}{c}\text { Síndrome de } \\
\text { Pierre Robin }+ \\
\text { polimalformativo }\end{array}$ \\
\hline Motivo de la distracción & $\begin{array}{l}\text { Apnea obstructiva grave. } \\
\text { Gastrostomía }\end{array}$ & $\begin{array}{l}\text { Apnea obstructiva grave. } \\
\text { Gastrostomía }\end{array}$ & Apnea obstructiva grave & $\begin{array}{l}\text { Apnea obstructiva grave. } \\
\text { Dificultad para la } \\
\text { alimentación }\end{array}$ \\
\hline Momento de realización & 1 año y 4 meses & 3,5 meses & 4 meses & 4,5 meses \\
\hline Velocidad de distracción & $1 \mathrm{~mm} /$ día & $1 \mathrm{~mm} /$ día & $1 \mathrm{~mm} /$ día & $1 \mathrm{~mm} /$ día \\
\hline Distracción total & $20 \mathrm{~mm}$ & $18 \mathrm{~mm}$ & $18 \mathrm{~mm}$ & $18 \mathrm{~mm}$ \\
\hline Retirada distractores & 8 semanas & 7 semanas & 8 semanas & 7 semanas \\
\hline Complicaciones & $\begin{array}{l}\text { Pérdida pin en el período de } \\
\text { consolidación. Infección } \\
\text { de la zona de inserción }\end{array}$ & $\begin{array}{l}\text { Dificultad de giro del } \\
\text { distractor }\end{array}$ & Ninguna & $\begin{array}{l}\text { Pérdida de un pin en el } \\
\text { período de distracción } \\
\text { activa (recolocación) }\end{array}$ \\
\hline
\end{tabular}

apnea obstructiva, aunque en 2 pacientes fue necesaria intubación intratraqueal durante un período largo antes de comenzar la distracción mandibular (tabla 1).

Todos ellos precisaron ingresos prolongados en la unidad de cuidados intensivos neonatales, hasta que fueron tratados con distracción osteogénica, debido a los valores bajos de la saturación de oxígeno y situación clínica muy lábil. El crecimiento y la ganancia ponderal fueron escasos. En dos casos se produjo desnutrición por imposibilidad para deglutir, lo que obligó a realizar gastrostomía en período neonatal.

La inducción anestésica fue difícil y la intubación se realizó mediante fibroscopia. En un paciente, después de varios intentos de intubación, no se consiguió realizar, por lo que se pospuso la cirugía una semana más tarde.

La distracción mandibular se comenzó 3 días después de la inserción de los distractores a un ritmo de $1 \mathrm{~mm} /$ día, 0,5 cada $12 \mathrm{~h}$. El rango de elongación conseguido fue entre 12 y $18 \mathrm{~mm}$ a cada lado. En todos los casos se sobrecorrigió, dejándolos prognatas, y en el niño afectado de síndrome de ThreacherCollins se produjo una mordida abierta anterior moderada (fig. 5). En el postoperatorio se les dejó un tubo intratraqueal que se retiró una vez desaparecieron los edemas posquirúrgicos, y transcurrió una semana del comienzo de la distracción, ya que la elongación mandibular conseguida hizo que se restablecieran los valores normales de saturación de oxígeno.

A medida que avanzaba la distracción ósea, el estado clínico de los niños fue mejorando llamativamente, comenzando a deglutir con normalidad, con la consiguiente ganancia ponderal.

Las complicaciones que surgieron fueron escasas: presencia de costras y serosidad en la piel alrededor de los clavos que se trató con pinceladas de povidona yodada.

En dos pacientes se extruyó uno de los pines, uno de ellos en la cuarta semana del período de contención y no se consideró reponerlo. En otro caso, se perdió en el período activo de distracción ósea y se reinsertó. En los pacientes no se observó lesión de la rama marginal del nervio facial.

Todos los pacientes presentaban fisura palatina. En tres de ellos se ha realizado reparación de la fisura palatina con veloplastia intravelar tras distracción osteogénica mandibular, sin ninguna complicación.

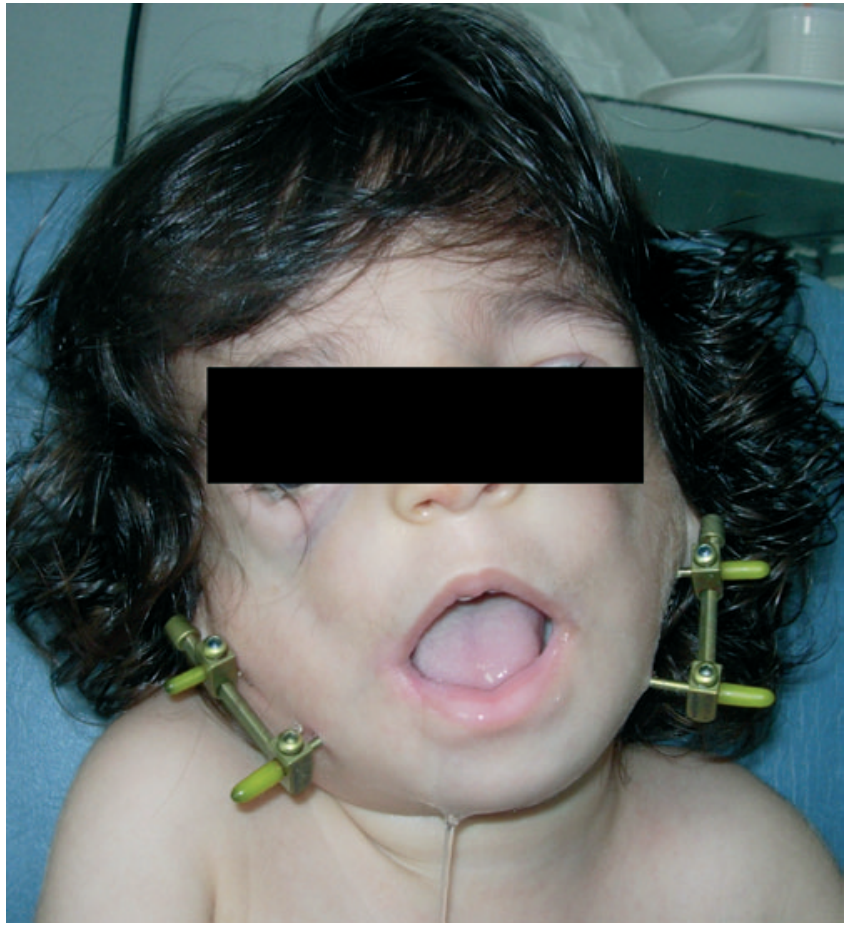

Figura 5 - Síndrome de Treacher-Collins. Sobrecorrección y mordida abierta anterior.

\section{Discusión}

Durante muchos años, a los pacientes con apnea obstructiva que no respondían a tratamiento postural se les realizaba una traqueostomía. Éste es un método efectivo en casos de apnea obstructiva, pero se asocia a una alta morbilidad, como traqueomalacia, bronquitis crónica, estenosis laríngea y riesgo de muerte debido a tapones de moco o extrusión/dislocación de tubo. También los pacientes a los que se realiza una traqueostomía requieren cuidados de enfermería y adiestramiento familiar.

Hay otros procedimientos, como por ejemplo: a) la glosopexia ${ }^{16}$, en la que la lengua se desplaza anteriormente y se fija a la mandíbula o al labio, con la intención de mantener 


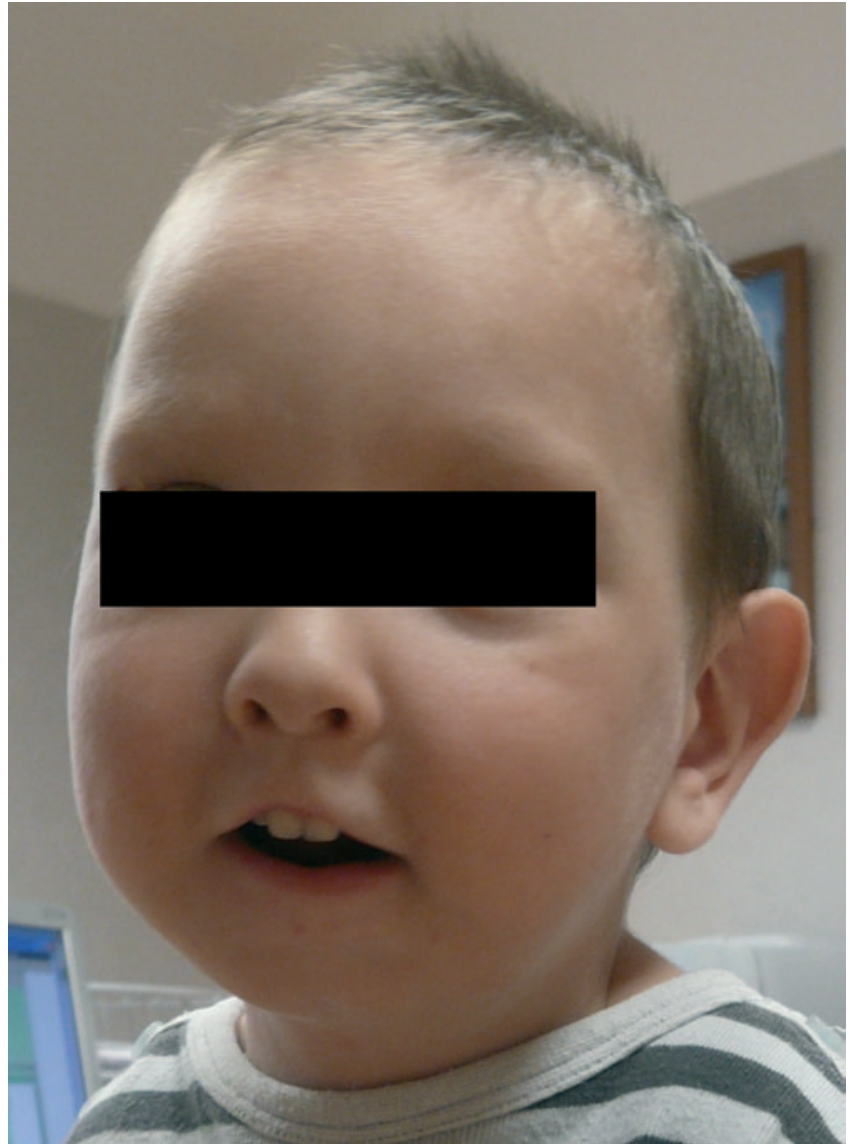

Figura 6 - Niño de la figura 3 a los 18 meses posdistracción.

permeable la vía aérea; b) la transfixión de la lengua con aguja de Kirschner ${ }^{17}$, situada anterior al ángulo mandibular, y c) la hiomandibulopexia, los cuales se pensaron para desplazar el hioides y la base de la lengua a una situación más anterior. No hay estudios que demuestren los beneficios de estas técnicas.

Muchos niños con micrognatia tienen un flujo de aire bajo, pero no como para impedir llevar una vida relativamente normal. Sin embargo, cuando estos pacientes presentan problemas, como infecciones de repetición de las vías respiratorias superiores, pueden desarrollar obstrucción respiratoria y requerir intubación intratraqueal. Estos pacientes tienen generalmente disminución en su curva de crecimiento y desarrollo, con una historia de problemas con la alimentación, peso insuficiente asociado a malnutrición, alta morbilidad pulmonar, largos períodos de hospitalización y problemas psicosociales y familiares.

La distracción osteogénica ${ }^{18}$ es una técnica eficaz de alargamiento mandibular en pacientes con hipoplasia mandibular. Los dispositivos para realizar la distracción pueden ser externos o internos. Estos últimos incluso pueden ser reabsorbibles ${ }^{19}$.

Esta técnica es una buena alternativa a la traqueostomía en pacientes neonatos con problemas de apnea obstructiva grave. Las indicaciones para realizar la distracción mandibular se van ampliando al mejorarse la técnica, por lo que se

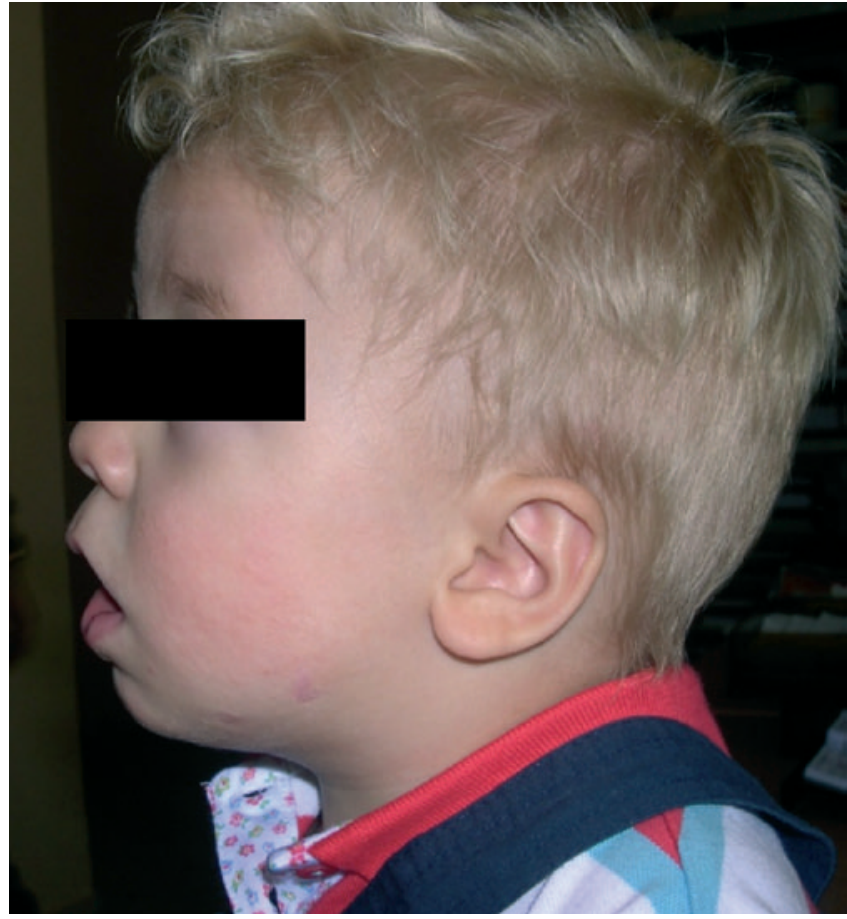

Figura 7 - Niño de la figura 4 a los 18 meses posdistracción.

benefician de este tratamiento algunos pacientes que no presentan una clínica respiratoria florida, pero sí otra sintomatología que mejora con la distracción ${ }^{20}$.

Junto con otros autores ${ }^{12}$, creemos que la polisomnografía puede ayudar a tomar la decisión de realizar la distracción mandibular, aunque también hay autores que no la consideran imprescindible ${ }^{21}$.

Nuestra experiencia es muy humilde, sólo 4 casos. En todos, el protocolo de distracción osteogénica que hemos seguido ha sido el descrito por Molina et $\mathrm{al}^{15}$.

En dos casos se extruyó uno de los pines. En uno, durante el período de contención, evaluamos la situación clínicamente y con controles radiológicos, y decidimos no volverlo a insertar, ya que la neoformación ósea en el área de distracción era visible. En otro caso, la pérdida fue muy temprana, durante el período de distracción activa y decidimos volverlo a insertar. Una semana después, y en el transcurso de esos días, se constató la recidiva y se produjo una asimetría en la hemimandíbula en la que hubo la pérdida del pin respecto al otro lado. El problema se solucionó volviendo a insertar el pin y distrayendo solamente en ese lado hasta conseguir simetría.

La estabilidad de los pines en niños tan pequeños es un problema, debido a la fragilidad y las dimensiones mínimas de la mandíbula. Para los próximos casos, nos planteamos utilizar los pines largos de Molina, un clavo único que atraviesa el cuerpo mandibular en ambos lados, combinado con clavos independientes a nivel del ángulo o bien dos clavos largos a nivel del cuerpo y del ángulo mandibular, para lograr la estabilidad del sistema de distracción y, simultáneamente, la sujeción de la musculatura de la lengua y suelo de boca. Así, en el período de distracción no sólo se elonga la mandíbula, también lo hacen los tejidos blandos, y se modifica la 
posición de la lengua, de ahí que la apnea obstructiva remita ${ }^{14}$

Un aspecto importante es el vector de distracción. En todos estos pacientes queremos obtener una elongación de rama y cuerpo mandibular simétrica en ambos lados y dirigida en sentido sagital paralelo al plano oclusal. A pesar de un cuidadoso planeamiento, en todos los casos no se logra el vector ideal, debido al problema técnico que suponen las pequeñas dimensiones de la mandíbula y a los esfuerzos para evitar lesiones de gérmenes dentarios. Al final del proceso de distracción, uno de los pacientes presentaba una mordida abierta anterior leve.

Los resultados estéticos también fueron excelentes (figs. 6 y 7) y mejoraron considerablemente los ángulos goniales, con una importante proyección anterior del mentón y una expansión considerable de los tejidos blandos del tercio inferior de la cara y de la porción superior del cuello, marcándose el ángulo cérvico-mandibular.

En un estudio publicado por Figueroa et $\mathrm{al}^{22}$ sobre el crecimiento mandibular en una serie de niños con síndrome de Pierre Robin no operados, los autores demuestran que la mandíbula efectivamente crece, pero no alcanza las dimensiones normales. Una pregunta que nos hacemos es, si después de la distracción ósea realizada en los neonatos, su mandíbula seguirá creciendo normalmente o, por el contrario, sigue creciendo poco debido a la información genética que lleva consigo. Sólo el seguimiento de los pacientes durante su crecimiento y desarrollo facial nos dará la respuesta. A su debido tiempo lo comunicaremos a la comunidad científica.

Respecto a las complicaciones de la distracción mandibular, son poco frecuentes, pero principalmente se han publicado alteraciones de la dentición permanente ${ }^{23}$.

Durante el seguimiento de la dentición decidua de los niños a los que hemos realizado distracción mandibular, destacaremos que, por el momento, se ha mantenido normal su cronología de la dentición. Las marcas cutáneas apenas son apreciables.

\section{Conclusiones}

La distracción mandibular temprana es la alternativa eficaz para evitar la traqueostomía en los niños con apnea obstructiva secundaria a hipoplasia mandibular grave, a la vez que mejora ostensiblemente la calidad de vida de los pacientes, ya que pueden incorporarse a su vida familiar sin riesgo vital inminente.

Con este método temprano de elongación mandibular, se ha agregado una valiosa herramienta de tratamiento, que resulta eficaz en todos los casos.

Por otra parte, hay la posibilidad de repetir el procedimiento si se necesitara posteriormente mayor elongación ósea. Los seguimientos a largo plazo han demostrado que este tratamiento no interfiere en el desarrollo de las piezas dentarias, ni en el crecimiento mandibular.

La distracción mandibular puede realizarse en recién nacidos, con lo que se resuelven los graves problemas ventilatorios, de intubación y mantenimiento de la vía aérea que presentan estos niños ${ }^{24}$.
Hay herramientas para el diagnóstico de certeza y de ayuda para la indicación del tratamiento, como son la exploración fibroscópica de las VADS y el estudio polisomnográfico.

\section{Conflicto de intereses}

Los autores declaran no tener conflicto de intereses.

\section{B I B L I O G R A F Í A}

1. Robin P. La chute de la base de la langue consideree comme une novelle cause de gene dans la respiration nasopharyngienne. Bull Acad Natl Med (Paris). 1923;89:37-41.

2. Lidsky ME, LanderTA, Sidman JD. Resolving Feeding Difficulties With Early Airway Intervention in Pierre Robin Sequence. Laryngoscope. 2008;118:120-3.

3. Sidman JD, Sampson D, Templeton B. Distraction osteogenesis of the mandible for airway obstruction in children. Laryngoscope. 2001;111:1137-46.

4. Shprintzen RJ. The implications of the diagnosis of Robin sequence. Cleft Palate Craniofac J. 1992;29:205-9.

5. Smith MC, Senders CW. Prognosis of airway obstruction and feeding difficulty in the Robin sequence. Int $J$ Pediatr Otorhinolaryngol. 2006;70:319-24.

6. Wagener S, Rayatt SS, Tatman AJ, Gornall P, Slator R. Management of infants with Pierre Robin sequence. Cleft Palate Craniofac J. 2003;40:180-5.

7. Caouette-Laberge L, Bayet B, Larocque Y. The Pierre Robin sequence: review of 125 cases and evolution of treatment modalities. Plast Reconstr Surg. 1994;93:934-42.

8. Schaefer RB, Stadler JA, 3rd, Gosain AK. To distract or not to distract: an algorithm for airway management in isolated Pierre Robin sequence. Plast Reconstr Surg. 2004;113:1113-25.

9. Sher AE. Mechanisms of airway obstruction in Robin sequence: implications for treatment. Cleft Palate Craniofac J. 1992;29:224-31.

10. Judge B, Hamlar D, Rimell FL. Mandibular distraction osteogenesis in a neonate. Arch Otolaryngol Head Neck Surg. 1999;125:1029-32.

11. McCarthy JG. The role of distraction osteogenesis in the reconstruction of the mandible in unilateral craniofacial microsomia. Clin Plast Surg. 1994;21:625-31.

12. Monasterio FO, Molina F, Berlanga F, Lopez ME, Ahumada H, Takenaga RH, et al. Swallowing disorders in Pierre Robin sequence: its correction by distraction. J Craniofac Surg. 2004;15:934-41.

13. Katzen JT, Holliday RA, McCarthy JG. Imaging the neonatal mandible for accurate distraction osteogenesis. J Craniofac Surg. 2001;12:26-30.

14. Monasterio FO, Drucker M, Molina F, Ysunza A. Distraction osteogenesis in Pierre Robin sequence and related respiratory problems in children. J Craniofac Surg. 2002;13:79-83.

15. Molina F, Ortiz Monasterio F, Yudocich M. La Microsomía Hemifacial y su tratamiento con Distracción ósea mandibular. Cir Plast Iberlatinamer. 2002;28:163-78.

16. Argamaso RV. Glossopexy for upper airway obstruction in Robin sequence. Cleft Palate Craniofac J. 1992;29:232-8.

17. Hadley RC, Johnson JB. Utilization of the Kirschner wire in Pierre Robin syndrome with case report. Plast Reconstr Surg. 1963;31:587-96.

18. Ilizarov GA. The principles of the Ilizarov method. Bull Hosp Jt Dis Orthop Inst. 1988;48:1-11. 
19. Burstein FD, Williams JK. Mandibular distraction osteogenesis in Pierre Robin sequence: application of a new internal singlestage resorbable device. Plast Reconstr Surg. 2005;115:61-7.

20. Rhee ST, Buchman SR. Pediatric mandibular distraction osteogenesis: the present and the future. J Craniofac Surg. 2003;14:803-8.

21. Fritz MA, Sidman JD. Distraction osteogenesis of the mandible. Curr Opin Otolaryngol Head Neck Surg. 2004;12:513-8.
22. Figueroa AA, Glupker TJ, Fitz MG, BeGole EA. Mandible, tongue, and airway in Pierre Robin sequence: a longitudinal cephalometric study. Cleft Palate Craniofac J .1991;28:425-34.

23. Parashar SY, Anderson PJ, David DJ. An unusual complication of mandibular distraction. Int J Paediatr Dent. 2006;16:55-8.

24. Tibesar RJ, Price DL, Moore EJ. Mandibular distraction osteogenesis to relieve Pierre Robin airway obstruction. Am J Otolaryngol. 2006;27:436-9. 